\title{
Quantifying Order during Field-driven Alignment of Colloidal Semi- conductor Nanorods
}

\author{
Rivi J. Ratnaweera, ${ }^{\dagger}$ Freddy A. Rodríguez Ortiz, ${ }^{\dagger}$ Nicholas J. Gripp ${ }^{\ddagger}$ and Matthew T. Sheldon ${ }^{\dagger \neq *}$ \\ ${ }^{\dagger}$ Department of Chemistry, Texas A\&M University, College Station Texas, 77843-3255, USA \\ ${ }^{\ddagger}$ Department of Materials Science \& Engineering, Texas A\&M University, College Station Texas, 77843-3255, USA
}

Supporting Information Placeholder

\begin{abstract}
Aligning large populations of colloidal nanorods (NRs) into ordered assemblies provides a strategy for engineering macroscopic functional materials with strong optical anisotropy. The bulk optical properties of such systems depend not only on the individual NR building blocks, but also on their meso- and macroscale ordering, in addition to more complex inter-particle coupling effects. Here, we investigate the dynamic alignment of colloidal CdSe/CdS NRs in the presence of AC electric fields by measuring concurrent changes in optical transmission. Our work identifies two distinct scales of interaction that give rise to the field-driven optical response: (1) the spontaneous mesoscale self-assembly of colloidal NRs into structures with increased optical anisotropy, and (2) the macroscopic ordering of NR assemblies along the direction of the applied AC field. By modeling the alignment of NR ensembles using directional statistics, we experimentally quantify the maximum degree of order in terms of the average deviation angle relative to the field axis. Results show a consistent improvement in alignment as a function of NR concentration-with a minimum average deviation of $18.7^{\circ}$ - indicating that mesoscale assembly helps facilitate field-driven alignment of colloidal NRs.
\end{abstract}

Colloidal semiconductor nanorods (SC NRs) have been widely studied as potential building blocks for optoelectronic devices ${ }^{1-7}$ due to their strong optical anisotropy, tunable band-edge emission and nearunity photoluminescence (PL) quantum yield. ${ }^{8-11}$ These onedimensional nanocrystals exhibit stronger quantum confinement ${ }^{12}$ perpendicular to their long axes, which modifies the spatial distribution of photoexcited charge carries, giving rise to pronounced optical anisotropy in the form of linearly polarized absorption and emission of light (see Figure 1). ${ }^{9,11}$. Recent advances in nanoparticle synthesis have further enabled precise control of size, composition, and morphology, affording tailored spectral- and angle-dependent absorption and emission patterns in these and related nanocrystalline materials..$^{13,14}$

The unique optical properties of individual NRs can be exploited at the macroscopic scale by assembling large populations of NRs into ordered superstructures. For instance, several studies have highlighted the promise of aligned anisotropic luminophores for application in luminescent solar concentrators ${ }^{4,5,15}$ and liquid crystal displays ${ }^{16,17}$. The ensemble properties of NR assemblies are determined not only by the individual NR building blocks, but also by their meso- and macroscale ordering, as well as more complex inter-particle coupling effects. ${ }^{18,19}$ Numerous techniques for collective alignment of NRs have been explored, ${ }^{20}$ and they can be broadly classified into two approaches: (1) taking advantage of the self-assembly of colloidal NRs into superlattic$\mathrm{es}^{21-24}$ and (2) applying external stimuli such as mechanical rubbing ${ }^{25}$, $\mathrm{AC}$ electric fields $\mathrm{s}^{26,27}$ or magnetic fields ${ }^{28}$ to promote orientation along a preferred axis.

To date, there has been significant progress studying the alignment of colloidal SC NRs in solution by AC electric fields as a strategy for producing functional materials with strong electro-optic modulation of optical anisotropy, typically in the $0.5-10 \mathrm{kHz}$ frequency regime. ${ }^{27,29}$ The underlying mechanism is based on torquing the electrostatic dipole moment in NRs-largest along the long axis-by means of an externally applied electric field, leading to collective alignment of NRs parallel to the field direction. ${ }^{27}$ Previous reports have attributed the electrostatic dipole moment in cadmium chalcogenide NRs to an intrinsic permanent dipole moment arising from their noncentrosymmetric wurtzite crystal structure. ${ }^{30}$ Additionally, in the presence of an external electric field, induced dipole moments may also contribute to the overall electrostatic dipole moment along the long axis. $^{27}$

The alternating magnitude of an applied AC field causes the NR ensemble to oscillate between aligned and randomly oriented configurations. Since NRs preferentially absorb light with polarization that is parallel to their long axes, switching between aligned and random configurations can give rise to a periodic change in absorbance, ${ }^{27,29,31}$ up to a relative change of $53 \%$ as demonstrated by Mohammadimasoudi et al. ${ }^{27}$ In a typical geometry (see Figure $2 \mathrm{a}$ ) where the sample is excited with light that is polarized perpendicular to the electric field, the absorbance of the sample decreases with increasing degree of alignment. This manifestation of the AC field-driven ordering in the timedependent absorbance signal has been used to quantify the alignment in a NR ensemble, with the highest possible degree of order targeted as most beneficial for optoelectronic applications.

Recent studies have reported partial ${ }^{29}$ and quasi-complete ${ }^{27}$ alignment of SC NRs in the presence of AC electric fields based on the fieldinduced relative change in absorbance. These reports, however, interpret data under the assumption that individual NRs are isolated in solution, and that the ensemble response can be attributed to the collective behavior of the individual, isolated NRs. 
(a)

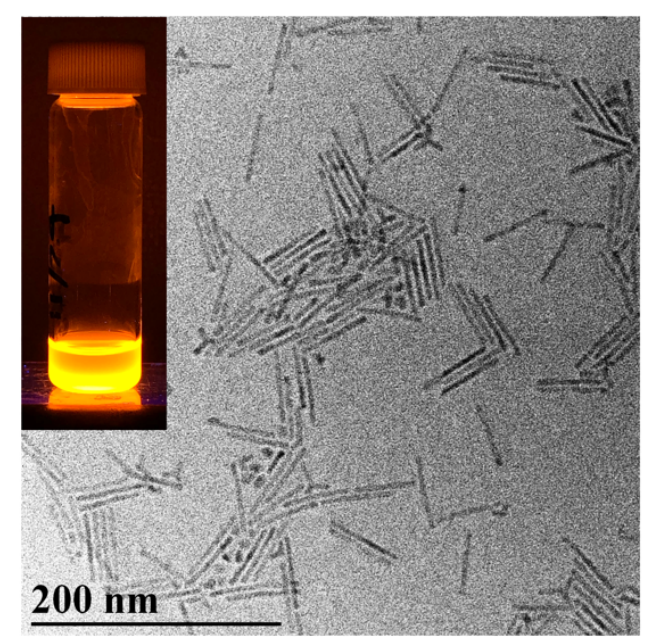

(b)

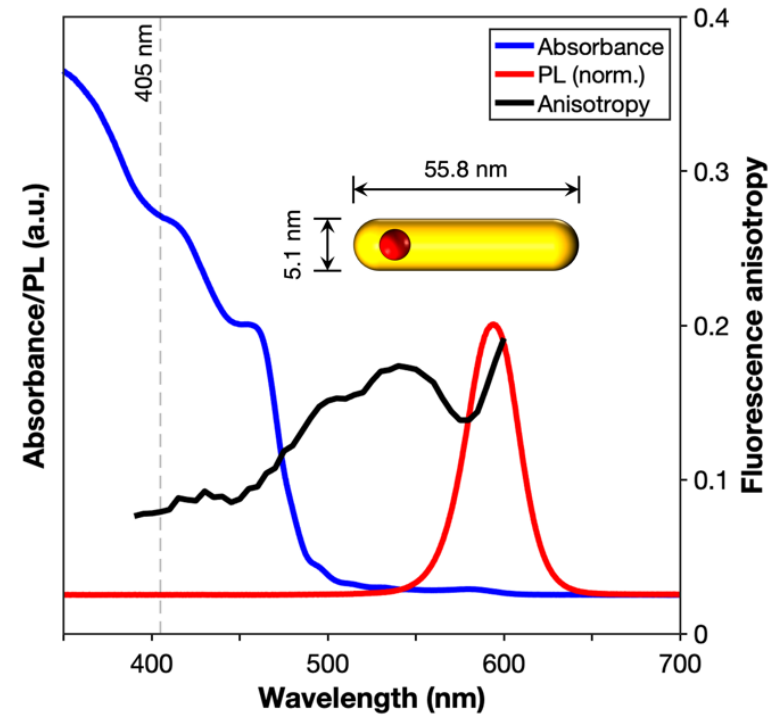

Figure 1. (a) Transmission electron micrograph (inset: PL emission of colloidal CdSe/CdS NRs). The average dimensions of the CdSe core $($ diameter $=2.3 \mathrm{~nm})$ and CdS shell $($ length $=55.8 \mathrm{~nm}$; diameter $=5.1$ $\mathrm{nm}$ ) were determined through statistical analysis (see Figure S2 in Supporting Information) of TEM images. (b) Absorbance, PL emission (normalized) and ensemble fluorescence anisotropy spectra of colloidal CdSe/CdS NRs.

Yet, growing evidence suggests that more complex interactions may be involved in the dynamic alignment of NRs. For example, colloidal SC NRs have been shown to spontaneously self-assemble into aggregated, microscale bundles in solution at concentrations as low as 1 $\mu \mathrm{M} .{ }^{28}$ Moreover, several previous studies indicate that the energetics associated with aligning individual NRs in an externally applied fieldup to field strengths that cause dielectric breakdown of the solvent system-minimally counteract the thermal energy of free rotation, if at all. Instead, synergistic effects between NRs may be partially responsible for the observed alignment. ${ }^{27,29}$ In this alternative picture, bundles or aggregates of NRs in solution are more polarizable by externally applied electrostatic fields compared to individual NRs, thereby enabling the pronounced field-driven response that is observed.

Solution phase self-assembly into bundles is also likely to modify the absorption anisotropy and other optical properties of colloidal NRs. For example, Pietra et al. observed increased linear dichroism when solutions of self-assembled NR bundles were placed in strong magnetic fields. ${ }^{28}$ The spontaneous aggregation of NRs into bundles-even in the absence of any external fields-complicates interpretations of ordering during optical measurements. Thus, ascertaining the optical anisotropy and related properties of the predominant NR species, i.e., isolated NRs or NR bundles, is critical for accurately quantifying the degree of field-driven ordering.

In this study, we investigate the alignment of colloidal CdSe/CdS core/shell NRs by probing the AC field-driven optical response. A statistical model based on a 3rd order von Mises-Fisher distribution ${ }^{32}$, i.e., a normal distribution describing 3-dimensional vector orientations, is used in conjunction with ensemble fluorescence anisotropy measurements to determine the average NR orientation relative to the direction of the applied field. We examine how varying the concentration of NRs impacts their electro-optic response and ensemble anisotropy to gain further insight into spontaneous mesoscale self-assembly.

An important insight from our study is the different scales of interaction that give rise to the macroscopic optical response. We see clear evidence for aggregation or self-assembly of NRs in solution, manifest as increased optical anisotropy compared to isolated NRs. Further, we observe that these self-assembled aggregates are more readily aligned by an external electrostatic field, giving rise to greater overall orientational order as well as stronger field-driven modulation of absorbance. Our approach highlights the contrasting electro-kinetic behavior of individual vs aggregated NRs and allows for quantitative determination of the degree of order using an optical analysis that accounts for these mesoscale self-assembly effects as well as the macroscopic field-driven behavior of the entire ensemble.

\section{Results and Discussion}

\section{Optical response of colloidal $\mathrm{CdSe} / \mathrm{CdS} \mathrm{NRs}$}

The field-driven alignment of colloidal CdSe/CdS core/shell NRs was investigated by measuring their time-dependent optical response in the presence of alternating (AC) electric fields. Dodecane-based dispersions of $\mathrm{CdSe} / \mathrm{CdS}$ NRs were loaded into $50 \mu \mathrm{m}$ thick sample cells (Figure S5 in Supporting Information) and exposed to uniform AC fields as depicted in Figure 2a. In order to avoid accumulation of NRs at the electrodes, a sufficiently high AC frequency $(2 \mathrm{kHz})$ was chosen, ensuring that the dynamic alignment of NRs occurs at a faster time scale compared to the cell transit time of any charged species. ${ }^{27}$ Concurrently, the time-dependent optical transmittance of the samples were monitored at $405 \mathrm{~nm}$ using a collimated light source and a photoreceiver (see Figure $2 \mathrm{a}$ ). The CdSe/CdS NRs used in this study were synthesized following a procedure ${ }^{33}$ described in literature, and the materials properties of samples were characterized using transmission electron microscopy (TEM), powder X-ray diffraction (XRD), and UV-vis spectroscopy (Figure 1), in addition to optical anisotropy measurements detailed below.

Figure $2 \mathrm{c}$ shows the transient optical response of a $2 \mu \mathrm{M}$ dispersion of NRs in the presence of a $\pm 7 \mathrm{~V} / \mu \mathrm{m}$ AC field. The transmittance of the sample fluctuates in response to the periodic alignment and relaxation via rotational diffusion of NRs. At zero field strength (1), the NRs are randomly oriented in solution, and the transmittance is at a minimum (maximum absorbance). However, as the field strength is gradually increased, the NRs begin to align parallel to the field direction (perpendicular to the polarization of incident laser beam), resulting in an overall decrease in absorbance and consequently an increase in transmittance. At peak field strength (2), the transmittance reaches a maximum, indicative of the system having reached a maximum degree of alignment for that magnitude of applied field. The rapid alignment and relaxation of NRs which occurs at a millisecond time scale can be perceived by the naked eye as a time-averaged increase in optical transmission (see Figure S9 in Supporting Information). 
(a)

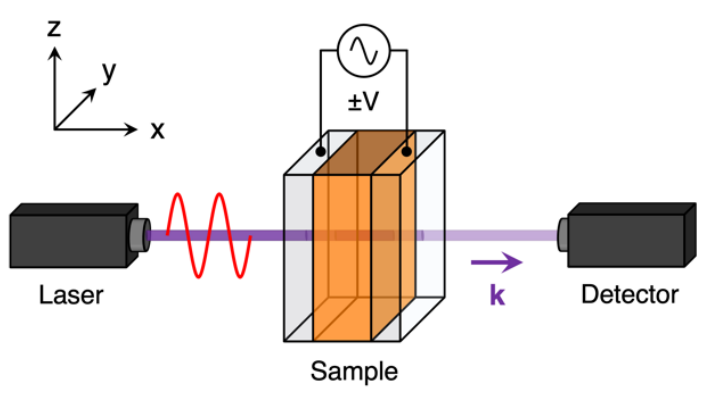

(b)

Random (V = 0)

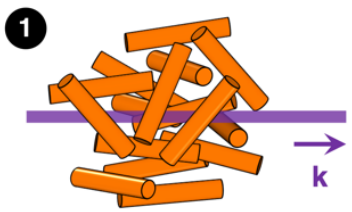

(c)
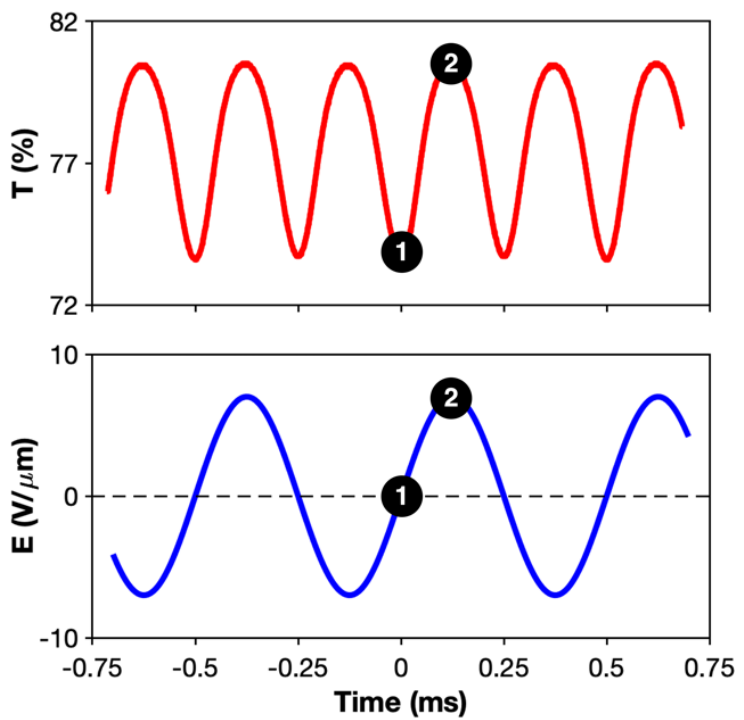

Figure 2. (a) Schematic representation of the experimental setup. (b) The alignment of NRs in the presence of an AC field when the magnitude of the applied voltage is equal to (1) zero and (2) the peak voltage. (c) Variation of applied field strength (bottom) and concurrent percent optical transmittance (top) as a function of time for a $2 \mu \mathrm{M}$ colloidal dispersion of CdSe/CdS NRs in the presence of a $2 \mathrm{kHz}, \pm 7$ $\mathrm{V} / \mu \mathrm{m}$ AC electric field.

The magnitude of the periodic absorbance shift is correlated with the degree of alignment in the NR ensemble: higher degrees of order result in larger shifts in absorbance. The relative change in absorbance $\left(\triangle A / A_{0}\right)$ has been proposed as a quantitative measure of ensemble alignment that is independent of external factors such as cell thickness, NR concentration or laser power. ${ }^{27} \mathrm{Eq} 1$ :

$$
\frac{\Delta A}{A_{0}}=\frac{A_{\text {peak }}-A_{0}}{A_{0}}
$$

describes $\Delta A / A_{0}$ in terms of the absorbance at peak voltage $\left(A_{\text {peak }}\right)$ and the absorbance when no external field is applied $\left(A_{0}\right)$.

The variation of $\Delta A / A_{0}$ was recorded as a function of field strength for five different NR concentrations (Figure 3). In each case, the mag- nitude of $\Delta A / A_{0}$ was found to increase with field strength, and saturate at approximately $15 \mathrm{~V} / \mu \mathrm{m}$, showing excellent agreement with previous studies. ${ }^{27}$ However, the limiting optical response exhibits a strong dependence on NR concentration: $\Delta A / A_{0}$ saturates at more negative values for samples with higher NR concentration, suggesting an overall improvement in ordering. We believe that the trend in $\Delta A / A_{0}$ can be attributed to inter-particle interactions that facilitate alignment, in particular, the spontaneous self-assembly of colloidal NRs into rafts or bundles, an effect that has been observed in similar colloidal systems. ${ }^{28}$ Increasing the NR concentration likely enhances the free-energy driving force for the formation of aggregates, resulting in an augmented electro-optic response. ${ }^{28}$

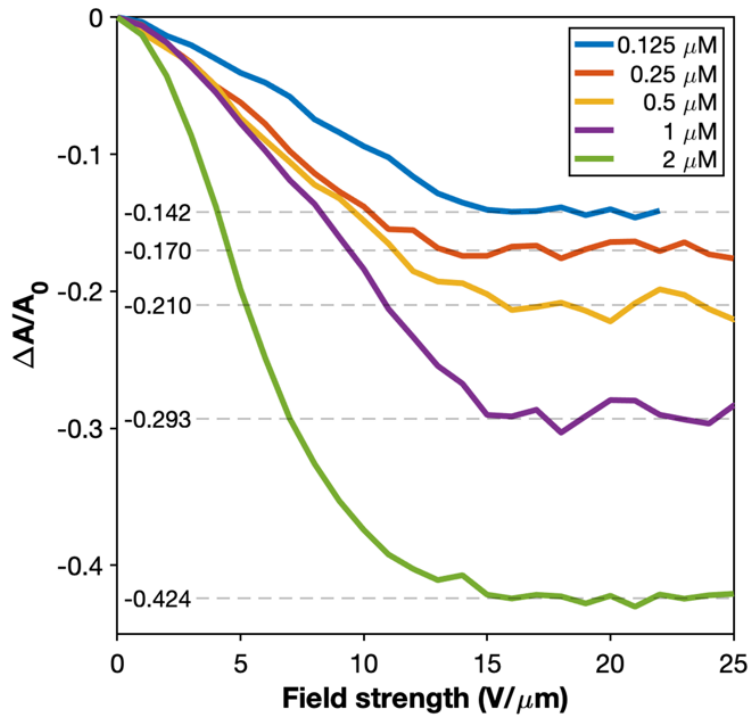

Figure 3. Variation of relative absorbance shift as a function of field strength measured for samples with different NR concentration.

It is important to note that the field-induced optical response of NRs depends not only on their ordering in solution, but also on the ensemble optical anisotropy of species in the colloidal dispersion. For example, given the same degree of ordering, NRs with a more ideal dipolar absorption and emission pattern would produce a larger shift in absorbance compared to NRs with a more isotropic absorption and emission pattern. Thus, quantitatively analyzing the optical anisotropy of the luminescent species is crucial for accurate determination of the overall degree of order.

\section{Ensemble optical anisotropy measurements}

The optical anisotropy of SC NRs is typically determined by measuring the polarization-dependent PL emission of individual NRs under a microscope $e^{9,11}$ or by performing ensemble anisotropy ${ }^{9-11}$ measurements of samples in solution. The latter strategy is generally favored as it samples the entire NR ensemble. ${ }^{9}$ Here, we measure the optical anisotropy of CdSe/CdS NRs using ensemble fluorescence anisotropy measurements. In a typical experiment, a random ensemble of NRs is excited by a vertically polarized source that selectively excites transition dipoles based on the projection of these dipoles parallel to the polarization axis of excitation. . $^{91,34,35}$ The PL emission from excited NRs is measured at $90^{\circ}$ relative to the excitation beam, through a vertical or horizontal polarizing filter. The ensemble anisotropy $(r)$ is defined according to eq 2 :

$$
r=\frac{I_{\|}-I_{\perp}}{I_{\|}+2 I_{\perp}}
$$


where $I_{\|}$and $I_{\perp}$ are the vertically and horizontally polarized emission intensities, respectively. ${ }^{34,35}$

The anisotropy of a random ensemble of emitters can, in theory, range between -0.2 and 0.4 depending on (1) the intrinsic dipole moment of the optical transition and (2) depolarization due to mismatch of excitation and emission dipole moments-typically caused by rotational diffusion between the excitation and emission events. The latter effect, while prominent in dichroic dyes ${ }^{34,35}$, can be regarded as negligible in SC NRs since the rotational diffusion rate $(>1 \mu \mathrm{s})$, even in lowviscosity solvents at room temperature, is significantly slow compared to the fluorescence lifetime $(10-50 \mathrm{~ns}){ }^{10}$ Dilute solutions of CdSe/CdS NRs have been experimentally shown to exhibit a peak anisotropy of $\sim 0.2$ for near band-edge excitation, and consistently lower values $(\sim 0.1)$ at higher energy excitation (Figure $2 c){ }^{9-11}$ Samples with no optical anisotropy show values of $r=0$, whereas an ensemble of randomly oriented, ideal dipoles show $r=0.4 .^{34,35}$

Figure 4 shows the ensemble anisotropy of CdSe/CdS NRs recorded as a function of NR concentration. The strong spectral dependence of anisotropy in SC NRs (Figure 2c) requires that the ensemble anisotropy measurements be carried out using the same excitation wavelength $(405 \mathrm{~nm})$ used in the $\Delta A / A_{0}$ measurements. As clearly apparent, the variation of ensemble anisotropy reveals a strong dependence on NR concentration. We observe two regimes of contrasting behavior for samples at low concentration or high concentration. In the dilute regime $(\leq 0.5 \mu \mathrm{M})$, the anisotropy increases with NR concentration. The positive correlation between anisotropy and concentration suggests that self-assembly of NRs may lead to the formation of mesoscale structures that have more ideal transition dipoles compared to individual NRs. This hypothesis concurs with proposed colloidal-phase NR assemblies ${ }^{28}$ in which NRs are aggregated in a side-by-side manner so that their long axes are co-aligned.

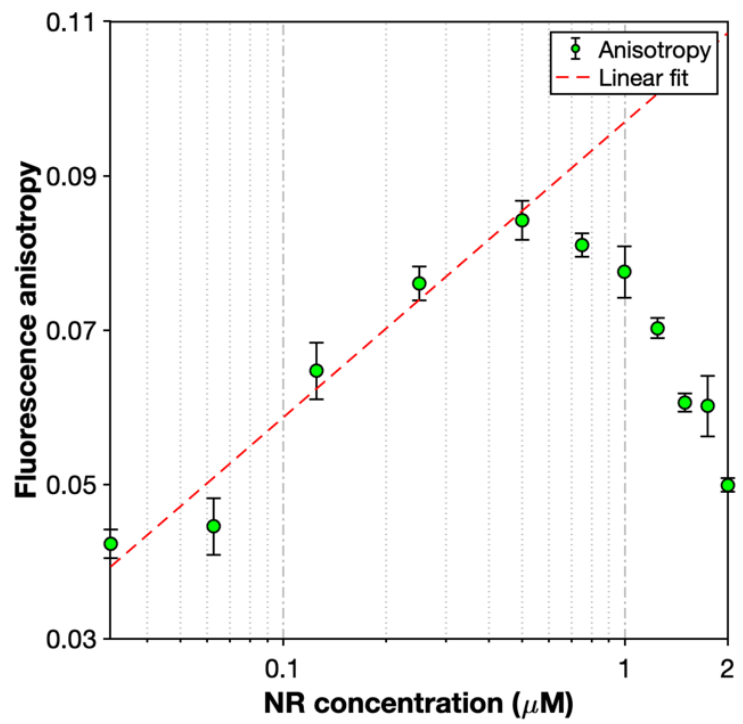

Figure 4. Ensemble fluorescence anisotropy of CdSe/CdS NRs at 405 $\mathrm{nm}$ excitation for samples with varying concentration (error bars represent the standard deviation). The dashed red line is the linear extrapolation of the data in the dilute concentration regime $(\leq 0.5 \mu \mathrm{M})$.

The decrease in anisotropy observed at higher concentrations can be attributed to multiple scattering and/or multiple reabsorption and reemission events, typical artifacts in ensemble anisotropy measurements when incident photons interact with more than one species before reaching the detector ${ }^{35}$ In order to estimate the anisotropy of the NR colloids independent of these artifacts intrinsic to the measurement, we have extrapolated the trend leading up to $0.5 \mu \mathrm{M}$ (dashed trace). This extrapolation aids further analysis provided below, though we acknowledge that this approximation is not a rigorous assessment of the true anisotropy, but instead, a rough estimate pertaining to the limiting case where the anisotropy continues to increase with increasing NR concentration. For simplicity, a linear trendline (on a logarithmic $\mathrm{x}$-axis) was chosen to extrapolate the data in the highconcentration regime.

\section{Theoretical model}

In order to translate the measured $\Delta A / A_{0}$ and ensemble anisotropy data into a quantitative measure of the orientational order, we developed an expression that predicts $\Delta A / A_{0}$, in terms of (1) the intrinsic anisotropy of the absorbing species and (2) the degree of field-driven macroscale ordering. The absorber could be an individual isolated NR or a self-assembled NR bundle. Our model simply assumes an arbitrary emitter/absorber with an intrinsic optical dipole.

The collective ordering of absorbers along the axis of the driving AC field is modeled using a 3rd order von Mises-Fisher distribution ${ }^{32}$. The probability $\left(f_{p}\right)$ of an absorber being oriented along an arbitrary direction $(\boldsymbol{r})$ can be expressed according to eq 3:

$$
f_{p}(\boldsymbol{r} ; \boldsymbol{\mu}, \kappa)=\frac{\kappa}{2 \pi\left(e^{\kappa}-e^{-\kappa}\right)} \exp \left(\kappa \boldsymbol{\mu}^{T} \boldsymbol{r}\right)
$$

where the parameters $\boldsymbol{\mu}$ and $\kappa$ represent, respectively, the direction of the externally applied field and the so-called focus factor. The parameter $\kappa$ can take on any value from 0 (randomly oriented ensemble of emitters) to infinity (perfectly aligned ensemble of emitters) and is directly correlated with the average deviation angle $(\bar{\theta})$ of the emitters relative to the unit vector $\boldsymbol{\mu}$ (see Figure S10 in Supporting Information).

According to Beer's law, the absorbance $(A)$ of a colloidal dispersion of nanoparticles can be expressed as shown in eq 4 :

$$
A=\left(\frac{N_{A}}{\ln 10}\right) \cdot \bar{\sigma} \cdot c \cdot l
$$

where $c$ is the concentration, $l$ is the path length, and $\bar{\sigma}$ is the average attenuation cross-section. ${ }^{36}$

This formalism can be adapted to calculate the absorbance of an ordered ensemble of NRs or NR bundles considering the angledependent absorption cross-section of individual absorbers. If each particle behaves as an ideal dipole absorber, the attenuation crosssection $(\sigma)$ of an arbitrarily oriented absorber excited by linearly polarized light (along the $\mathrm{X}-\mathrm{Z}$ plane in Figure 5) is related to its zenith $(\theta)$ and azimuth $(\varphi)$ angles according to eq 5 :

$$
\sigma(\theta, \varphi)=k \sin ^{2} \theta \cos ^{2} \varphi
$$

where $k$ represents a proportionality constant.

The average attenuation cross-section of an ensemble $(\bar{\sigma})$ can be calculated according to eq 6 :

$$
\bar{\sigma}(\kappa)=\int_{0}^{2 \pi} \int_{0}^{\pi} f_{p}(\theta, \varphi) \sigma(\theta, \varphi) d \theta d \varphi
$$

by integrating the single-particle attenuation cross-section $(\sigma)$ weighted by the normalized probability density of orientations $\left(f_{p}\right)$. The angles $\theta$ and $\varphi$ represent the zenith and azimuth angles of an arbitrary absorber, respectively. 


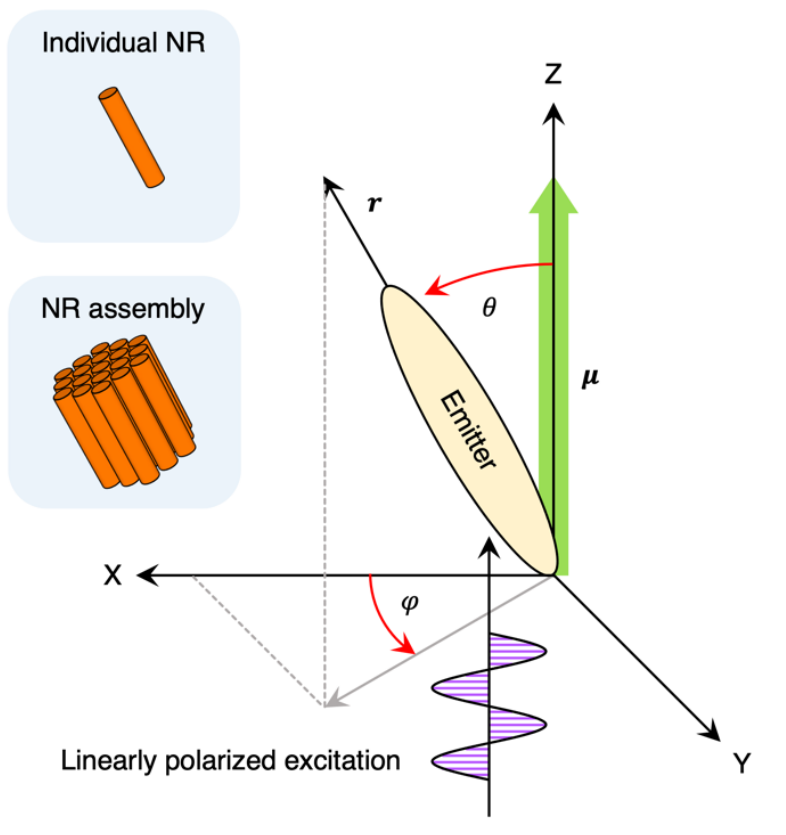

Figure 5. The orientation of an arbitrary emitter $(r)$ and applied field direction $(\mu)$ defined in terms of zenith $(\theta)$ and azimuth $(\varphi)$ angles based on a Cartesian coordinate system. The axis of optical excitation polarization lies along the $\mathrm{X}-\mathrm{Z}$ plane. The emitter could be an individual isolated NR or a self-assembled NR bundle (inset).

Combining eq 4 and eq 6 yields Equation 7:

$$
A=\left(\frac{N_{A} c l}{\ln 10}\right) \int_{0}^{2 \pi} \int_{0}^{\pi} f_{p}(\theta, \varphi) \sigma(\theta, \varphi) d \theta d \varphi
$$

which describes the ensemble absorbance $(A)$ in terms of concentration $(c)$, path length $(l)$ and single-particle cross section $(\sigma)$.

Substituting for absorbance in eq 1 negates the dependence on concentration and path length, resulting in eq 8 :

$$
\frac{\Delta A}{A_{0}}=\frac{\bar{\sigma}(\kappa)-\bar{\sigma}(\kappa=0)}{\bar{\sigma}(\kappa=0)}
$$

which can be used to calculate the relative change in absorbance $\left(\Delta A / A_{0}\right)$ as a function of the focus factor $(\kappa)$.

The predicted relative absorbance shift decreases with increasing order and saturates at -1 as the system approaches complete alignment $(\bar{\sigma}(\kappa) \rightarrow 0)$. This behavior can be explained by the complete absence of an absorption cross-section for ideal dipole absorbers when oriented perpendicular to the polarization of excitation.

While NRs exhibit strong linearly polarized absorption and emission, their optical behavior deviates from those of ideal dipoles, in part, due to features of their band structure. ${ }^{9}$ Previous studies have treated this non-ideal optical behavior by considering a linear combination of isotropic and dipole character. ${ }^{4,5}$ As such, the expression for singleparticle absorption cross-section (eq 5 ) is modified according to eq 9:

$$
\sigma(\theta)=k\left[\beta \sin ^{2} \theta \cos ^{2} \varphi+(1-\beta)\right]
$$

where $\beta(0 \leq \beta \leq 1)$ is a linear interpolation parameter which describes the dipole character of an individual emitter, i.e., how closely its absorption/emission resembles that of an ideal dipole $(\beta=1)$ or an isotropic emitter $(\beta=0)$.

The fluorescence anisotropy of a random ensemble of ideal dipole emitters is conventionally calculated by integrating the excitationweighted contributions of vertically and horizontally polarized emission intensities over the full range of emitter orientations. ${ }^{35}$ Building from this approach, we have derived (see Supporting Information) eq 10:

$$
r=\frac{2 \beta^{2}}{\left(20 \beta^{2}-60 \beta+45\right)}
$$

which describes the fluorescence anisotropy $(r)$ of a random ensemble of non-ideal dipole emitters in terms of the intrinsic dipole character $(\beta)$ of individual emitters. ${ }^{5}$

The dipole character of NRs or NR bundles in the samples studied above can be calculated by substituting experimentally measured ensemble anisotropy values in Equation 10. Then, the correlation between $\Delta A / A_{0}$ and $\kappa$ (Equation 8 ) is used to determine the degree of order in each sample studied in terms of the average deviation angle $(\bar{\theta})$. Table 1 summarizes the limiting relative change in absorbance, ensemble anisotropy and degree of alignment for each sample. For NR concentrations above $0.5 \mu \mathrm{M}$, we have also included estimates of the average deviation angles by inputting the extrapolated ensemble anisotropy data (dashed trace in Figure 4). We also report the statistical standard deviation (SD) of the fitted and extrapolated deviation angles.

In the regime where anisotropy measurements are free of scattering artifacts $(\leq 0.5 \mu \mathrm{M})$, the average deviation angle decreases with increasing NR concentration, suggesting that macroscopic field-driven ordering improves as the degree of mesoscale self-assembly is increased. That is, it appears that NR aggregates align more easily, and order more completely compared to individual isolated NRs.

At higher concentrations $(>0.5 \mu \mathrm{M})$, the dipolar character of emitters, $\beta$, is likely underestimated due to depolarization caused by scattering and/or reabsorption during the ensemble anisotropy measurements, as discussed above. This effect may result in somewhat exaggerated apparent degrees of macroscale alignment by our analysis, because larger fitted values for $\kappa$ are required to match the experimentally measured $\Delta A / A_{0}$. In other words, the measured magnitude of $\Delta A / A_{0}$ could have resulted from either more ideal dipole emitters undergoing weaker ordering, or less-ideal dipole emitters undergoing stronger ordering. To correct for this potential complication, we also calculate average deviation angles using the extrapolated ensemble anisotropy data (see dashed red line in Figure 4). As summarized in the last two columns of Table 1, this correction increases the fitted average deviation angles. However, the degree of order is still observed to increase as a function of NR concentration.

Finally, we note that we have also performed studies to promote the formation of NR bundles by adding a varying amount of anti-solvent that decreases colloidal stability (see Figure S8 in Supporting Information). Our results are consistent with the dependence on NR concentration discussed above: the ensemble anisotropy increases with increasing concentration of anti-solvent (decreasing colloidal stability).

Overall, these results support our hypothesis that the electro-optic response arises due to separate contributions from (1) the optical anisotropy of mesoscale NR assemblies that form in the absence of applied fields and (2) macroscopic ordering imposed by the electrostatic forces of the driving AC field. The greater magnitude of $\Delta A / A_{0}$ at higher NR concentrations is attributed both to an increase in the optical anisotropy of the emitting species, i.e., the formation of NR bundles, as well as a greater susceptibility of these bundles to field-driven ordering. While the magnitude of the experimentally measured $\Delta A / A_{0}$ in our study is comparable with previous reports ${ }^{27}$, our analysis indicates a significantly lower degree of maximum NR alignment. The disparity lies in our analysis of the role of individual absorbers. Instead of assuming colloidal NRs to be individual isolated particles, we treat them as aggregated bundles with varying degrees of characteristic concentration-dependent optical anisotropy. 
Table 1. Limiting $\Delta \mathbf{A} / \mathbf{A}_{0}$, Ensemble Fluorescence Anisotropy and Average Deviation Angle at Maximum Alignment.

\begin{tabular}{|c|c|c|c|c|c|}
\hline $\begin{array}{c}\text { NR Concentration } \\
(\mu \mathrm{M})\end{array}$ & Limiting $\Delta A / A_{0}$ & \multicolumn{2}{|c|}{ Ensemble Anisotropy } & \multicolumn{2}{|c|}{ Average Deviation Angle, $\bar{\theta} \pm \operatorname{SD}\left({ }^{\circ}\right)$} \\
\hline 0.125 & -0.142 & 0.0647 & $\mathrm{~N} / \mathrm{A}$ & $41.9 \pm 23.6$ & $\mathrm{~N} / \mathrm{A}$ \\
\hline 0.25 & -0.170 & 0.0760 & $\mathrm{~N} / \mathrm{A}$ & $39.8 \pm 22.3$ & $\mathrm{~N} / \mathrm{A}$ \\
\hline 0.5 & -0.210 & 0.0842 & $\mathrm{~N} / \mathrm{A}$ & $36.2 \pm 20.1$ & $\mathrm{~N} / \mathrm{A}$ \\
\hline 1 & -0.293 & 0.0775 & 0.0969 & $26.2 \pm 14.1$ & $29.5 \pm 16.0$ \\
\hline
\end{tabular}

\section{Conclusions}

We have demonstrated AC field-driven mechanical alignment of colloidal $\mathrm{CdSe} / \mathrm{CdS}$ NRs by measuring concurrent changes in optical transmission. Our work identifies two distinct scales of interaction that give rise to the macroscopic optical response: (1) the spontaneous mesoscale self-assembly of colloidal NRs into bundles with greater optical anisotropy than ensembles of individual NRs, and (2) the macroscale ordering of $\mathrm{NR}$ assemblies along the direction of the applied driving field. Ensemble optical anisotropy measurements show that aggregated $\mathrm{NR}$ assemblies are more anisotropic compared to isolated NRs, and therefore, provide an enhanced electro-optic response. A key insight from our study is that the field-driven relative change in absorbance depends not only on the degree of NR alignment, but also on the optical anisotropy of the absorbing species-i.e., isolated NRs or NR assemblies. By analyzing our measurements using an optical model that we derived, we have quantified the degree of order in terms of the average emitter orientation relative to the field axis. Our results show a consistent improvement in macroscopic alignment as a function of $\mathrm{NR}$ concentration, with a minimum average deviation angle of $18.7^{\circ}$ based on our extrapolated values for anisotropy. Our results also indicate that NR bundles are more responsive to field-driven alignment compared to individual NRs. This work provides a basis for quantitative determination of AC field-driven ordering in colloidal NRs and may further aid the design and fabrication of optoelectronic devices with aligned anisotropic nanocrystals.

\section{Methods}

Materials. Cadmium oxide (CdO, $\geq 99.99 \%$ trace metals basis), Selenium (99.99\% trace metals basis), Sulfur (99.998\% trace metals basis), Octadecylphosphonic acid (ODPA, 97\%), Hexylphosphonic acid (95\%), Trioctylphosphine oxide (TOPO, ReagentPlus, 99\%), Trioctylphosphine (TOP, 97\%), 1-octadecene (ODE, technical grade 90\%), hexanes (anhydrous mixture of isomers, 99\%), methanol (anhydrous, 99.8\%) were purchased from Sigma Aldrich.

Preparation of CdSe/CdS NRs. Colloidal CdSe/CdS NRs were synthesized using a seeded growth method previously described by Carbone et. al. ${ }^{33}$ The as-synthesized nanorods were precipitated with the addition of methanol as an anti-solvent and purified by centrifugation at 3000 RCF for 8 minutes. The resulting precipitate was further cleaned by resuspending in a mixture of hexane and methanol followed by centrifugation at 3000 RCF for 8 minutes. This purification step was repeated twice before the final precipitate was resuspended in $10 \mathrm{~mL}$ of anhydrous hexane and stored under argon for further analysis.

Characterization. High-resolution transmission electron microscopy (HR-TEM) images were collected using an FEI Tecnai G2 F20 ST FE-TEM operated at an accelerating voltage of $200 \mathrm{kV}$. Absorbance and PL spectra were collected on an Ocean Optics Flame-S-UV-Vis spectrometer with an Ocean Optics DH-2000-BAL deuterium and halogen lamp as the light source. Powder X-ray diffraction (XRD) measurements were performed using a Bruker-AXS D8 Advanced Bragg-Brentano diffractometer with a $\mathrm{Cu} \mathrm{Ka}$ radiation source $(\lambda=$ $1.5418 \AA$ ).

Optical response measurements. Sinusoidal AC electric fields were applied across colloidal dispersions of CdSe/CdS NRs using a function generator (RIGOL DG1032) coupled with a high-voltage amplifier (Trek Model 2220). Concurrent changes in optical transmission of a $405 \mathrm{~nm}$ laser (RGBLase FBB-405-200-FS-C-1-0) were measured using a photoreceiver (2007 Nirvana Auto-Balanced Optical Receiver) and a digital oscilloscope (RIGOL DS2302A). Sample cells were prepared by adhering pairs of patterned ITO glass slides (MSE Supplies) using a mixture of optical adhesive (Norland NOE81) and $50 \mu \mathrm{m}$ spacer beads (Cospheric). Detailed schematics of the experimental setup and sample cell design are included in the Supporting Information.

Fluorescence anisotropy measurements. The ensemble fluorescence anisotropy of colloidal CdSe/CdS NRs was measured using a home-built L-format optical setup (see Figure S6 in Supporting Information) equipped with a $405 \mathrm{~nm}$ laser (RGBLase FBB-405-200-FS-C1-0). The excitation beam was attenuated using a variable neutral density filter (NDC-25C-2) and polarized through a hand-mounted GlanTaylor polarizer (Thorlabs GT15-A). The PL emission from the sample was collected at $90^{\circ}$ relative to the excitation beam using coaligned irises (Thorlabs ID25) and polarized using a second Glan-Taylor polarizer (Thorlabs GT15-A) before being directed to a free-space biased Si Photodetector (Thorlabs DET100A2).

\section{ASSOCIATED CONTENT}

\section{Supporting Information}

The Supporting Information is available free of charge on the ACS Publications website.

TEM images and XRD pattern; schematics describing experimental setups for measuring time-dependent absorbance and ensemble fluorescence anisotropy; additional optical response and ensemble anisotropy data measured using an alternate batch of CdSe/CdS NRs; overhead images of UV-illuminated sample cell with and without an external AC field; plot of average deviation angle vs focus factor $(\kappa)$; derivation of theoretical expression for ensemble fluorescence anisotropy (PDF)

\section{AUTHOR INFORMATION}

\section{Corresponding Author}

Matthew T. Sheldon - Department of Chemistry, Texas A\&M University, College Station Texas, 77843-3255, USA; Email: sheldonm@tamu.edu

\section{Author Contributions}


M.T.S. and R.J.R. conceived the idea. R.J.R., F.A.R.O. and N.J.G. designed and conducted the experiments. R.J.R. and F.A.R.O. performed characterization. R.J.R. derived the theoretical equations. R.J.R., F.A.R.O. and M.T.S. wrote the manuscript. All authors reviewed the manuscript.

\section{Notes}

The authors declare no competing financial interests.

\section{ACKNOWLEDGMENTS}

This work was supported by the Gordon and Betty Moore Foundation (Grant GBMF6882), the Welch Foundation (A-1886), and the Air Force Office of Scientific Research (FA9550-16-1-0154). The authors would like to thank Je-Ruei Wen for assistance with TEM imaging as well as Ryan Kutayiah and Benjamin Roman for invaluable discussion.

\section{REFERENCES}

1. Bronstein, N. D.; Li, L.; Xu, L.; Yao, Y.; Ferry, V. E.; Alivisatos, A. P.; Nuzzo, R. G. Luminescent Solar Concentration with Semiconductor Nano rods and Transfer-Printed Micro-Silicon Solar Cells. ACS Nano 2014, 8, 44-53.

2. Cunningham, P. D.; Souza, J. B.; Fedin, I.; She, C.; Lee, B.; Talapin, D. V. Assessment of Anisotropic Semiconductor Nanorod and Nanoplatelet Heterostructures with Polarized Emission for Liquid Crystal Display Technology. ACS Nano 2016, 10, 5769-5781.

3. Deka, S.; Quarta, A.; Lupo, M. G.; Falqui, A.; Boninelli, S.; Giannini, C.; Morello, G.; De Giorgi, M.; Lanzani, G.; Spinella, C.; Cingolani, R.; Pellegrino, T.; Manna, L. CdSe/CdS/ZnS Double Shell Nanorods with High Photoluminescence Efficiency and Their Exploitation As Biolabeling Probes. J. Am. Chem. Soc. 2009, 131, 2948-2958.

4. Fisher, M.; Farrell, D.; Zanella, M.; Lupi, A.; Stavrinou, P. N.; Chatten, A. J. Utilizing vertically aligned $\mathrm{CdSe} / \mathrm{CdS}$ nanorods within a luminescent solar concentrator. Appl. Phys. Lett. 2015, 106, 041110.

5. Martin, J.; Ratnaweera, R.; Kumar, S.; Wen, J.-R.; Kutayiah, A. R. Sheldon, M. Detailed balance efficiencies for luminescent solar concentrators with aligned semiconductor nanorods: the benefits of anisotropic emission. J. Photonics Energy 2020, 10, 025501.

6. Pisanello, F.; Martiradonna, L.; Spinicelli, P.; Fiore, A.; Hermier, J. P.; Manna, L.; Cingolani, R.; Giacobino, E.; De Vittorio, M.; Bramati, A. Dots in rods as polarized single photon sources. Superlattices Microstruct. 2010, 47, 165-169.

7. Rizzo, A.; Nobile, C.; Mazzeo, M.; De Giorgi, M.; Fiore, A.; Carbone, L.; Cingolani, R.; Manna, L.; Gigli, G. Polarized Light Emitting Diode by Long-Range Nanorod Self-Assembling on a Water Surface. ACS Nano 2009, 3, 1506-1512.

8. Coropceanu, I.; Rossinelli, A.; Caram, J. R.; Freyria, F. S.; Bawendi, M. G. Slow-Injection Growth of Seeded CdSe/CdS Nanorods with Unity Fluorescence Quantum Yield and Complete Shell to Core Energy Transfer. ACS Nano 2016, 10, 3295-3301.

9. Diroll, B. T.; Dadosh, T.; Koschitzky, A.; Goldman, Y. E.; Murray, C. B. Interpreting the Energy-Dependent Anisotropy of Colloidal Nanorods Using Ensemble and Single-Particle Spectroscopy. J. Phys. Chem. C 2013, 117, 23928-23937.

10. Diroll, B. T.; Koschitzky, A.; Murray, C. B. Tunable Optical Anisotropy of Seeded CdSe/CdS Nanorods. J. Phys. Chem. Lett. 2014, 5, 85-91.

11. Hadar, I.; Hitin, G. B.; Sitt, A.; Faust, A.; Banin, U. Polarization Properties of Semiconductor Nanorod Heterostructures: From Single Particles to the Ensemble. J. Phys. Chem. Lett. 2013, 4, 502-507.

12. Krahne, R.; Morello, G.; Figuerola, A.; George, C.; Deka, S.; Manna, L. Physical properties of elongated inorganic nanoparticles. Phys. Rep. 2011, 501, 75-221.

13. Jing, L.; Kershaw, S. V.; Li, Y.; Huang, X.; Li, Y.; Rogach, A. L.; Gao, M. Aqueous Based Semiconductor Nanocrystals. Chem. Rev. 2016, 116, 10623 10730.

14. Ussembayev, Y. Y.; Hens, Z.; Neyts, K. Contrasting Anisotropy of Light Absorption and Emission by Semiconductor Nanoparticles. ACS Photonics 2019, 6, 1146-1152.

15. Verbunt, P. P. C.; de Jong, T. M.; de Boer, D. K. G.; Broer, D. J.; Debije, M. G. Anisotropic light emission from aligned luminophores. Eur. Phys. J. Appl. Phys. 2014, 67, 10201.
16. Li, L.-S.; Alivisatos, A. P. Semiconductor Nanorod Liquid Crystals and Their Assembly on a Substrate. Adv. Mater. 2003, 15, 408-411.

17. Li, L.-s.; Walda, J.; Manna, L.; Alivisatos, A. P. Semiconductor Nanorod Liquid Crystals. Nano Lett. 2002, 2, 557-560.

18. Vanmaekelbergh, D. Self-assembly of colloidal nanocrystals as route to novel classes of nanostructured materials. Nano Today 2011, 6, 419-437.

19. Kutayiah, A. R.; Kumar, S.; Ratnaweera, R.; Easwaran, K.; Sheldon, M. Markov chains for modeling complex luminescence, absorption, and scattering in nanophotonic systems. Opt. Express 2021, 29, 4249-4269.

20. Ramasamy, K.; Gupta, A. Routes to self-assembly of nanorods. J. Mater. Res. 2013, 28, 1761-1776.

21. Baker, J. L.; Widmer-Cooper, A.; Toney, M. F.; Geissler, P. L.; Alivisatos, A. P. Device-Scale Perpendicular Alignment of Colloidal Nanorods. Nano Lett. 2010, 10, 195-201.

22. Querner, C.; Fischbein, M. D.; Heiney, P. A.; Drndić, M. Millimeter-Scale Assembly of CdSe Nanorods into Smectic Superstructures by Solvent Drying Kinetics. Adv. Mater. 2008, 20, 2308-2314.

23. Talapin, D. V.; Shevchenko, E. V.; Murray, C. B.; Kornowski, A.; Förster, S.; Weller, H. CdSe and CdSe/CdS Nanorod Solids. J. Am. Chem. Soc. 2004, 126, 12984-12988.

24. Zanella, M.; Gomes, R.; Povia, M.; Giannini, C.; Zhang, Y.; Riskin, A.; Van Bael, M.; Hens, Z.; Manna, L. Self-Assembled Multilayers of Vertically Aligned Semiconductor Nanorods on Device-Scale Areas. Adv. Mater. 2011, 23, 2205-2209.

25. Amit, Y.; Faust, A.; Lieberman, I.; Yedidya, L.; Banin, U. Semiconductor nanorod layers aligned through mechanical rubbing. Phys. Status Solidi A 2012, 209, 235-242.

26. Hu, Z.; Fischbein, M. D.; Querner, C.; Drndić, M. Electric-FieldDriven Accumulation and Alignment of CdSe and CdTe Nanorods in $\mathrm{Na}$ noscale Devices. Nano Lett. 2006, 6, 2585-2591.

27. Mohammadimasoudi, M.; Hens, Z.; Neyts, K. Full alignment of dispersed colloidal nanorods by alternating electric fields. RSC Adv. 2016, 6, 55736-55744.

28. Pietra, F.; Rabouw, F. T.; van Rhee, P. G.; van Rijssel, J.; Petukhov, A. V.; Erné, B. H.; Christianen, P. C. M.; de Mello Donegá, C.; Vanmaekelbergh, D. Self-Assembled CdSe/CdS Nanorod Sheets Studied in the Bulk Suspension by Magnetic Alignment. ACS Nano 2014, 8, 10486-10495.

29. Kamal, J. S.; Gomes, R.; Hens, Z.; Karvar, M.; Neyts, K.; Compernolle, S.; Vanhaecke, F. Direct determination of absorption anisotropy in colloidal quantum rods. Phys. Rev. B 2012, 85, 035126.

30. Nann, T.; Schneider, J. Origin of permanent electric dipole moments in wurtzite nanocrystals. Chem. Phys. Lett. 2004, 384, 150-152.

31. Rossi, D.; Han, J. H.; Jung, W.; Cheon, J.; Son, D. H. Orientational Control of Colloidal 2D-Layered Transition Metal Dichalcogenide Nanodiscs via Unusual Electrokinetic Response. ACS Nano 2015, 9, 8037-8043.

32. Mardia, K. V.; Jupp, P. E. Tests on von Mises Distributions. In Directional Statistics; 1999; pp 119-142.

33. Carbone, L.; Nobile, C.; De Giorgi, M.; Sala, F. D.; Morello, G.; Pompa, P.; Hytch, M.; Snoeck, E.; Fiore, A.; Franchini, I. R.; Nadasan, M.; Silvestre, A. F.; Chiodo, L.; Kudera, S.; Cingolani, R.; Krahne, R.; Manna, L. Synthesis and Micrometer-Scale Assembly of Colloidal CdSe/CdS Nanorods Prepared by a Seeded Growth Approach. Nano Lett. 2007, 7, 2942-2950.

34. Ameloot, M.; vandeVen, M.; Acuña, A. U.; Valeur, B. Fluorescence anisotropy measurements in solution: Methods and reference materials (IUPAC Technical Report). Pure Appl. Chem. 2013, 85, 589-608.

35. Lakowicz, J. R. Fluorescence Anisotropy. In Principles of Fluorescence Spectroscopy; Springer US: Boston, 2006; pp 291-319.

36. Skoog, D. A.; West, D. M.; Holler, F. J.; Crouch, S. R. Fundamentals of Analytical Chemistry; Cengage Learning: 2013. 
For Table of Contents/Abstract Only

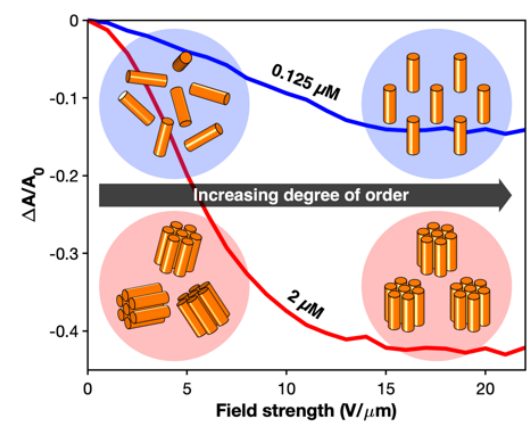

